\title{
STANDARD MODEL AND FLAVOR PHYSICS MEASUREMENTS WITH THE ATLAS DETECTOR*
}

\author{
PAWEe MALECKI \\ on behalf of the ATLAS Collaboration \\ The Henryk Niewodniczański Institute of Nuclear Physics PAN \\ Radzikowskiego 152, 31-342 Kraków, Poland
}

(Received April 24, 2014)

\begin{abstract}
This article presents recent results obtained by the ATLAS experiment at the Large Hadron Collider (LHC) regarding the measurements of Standard Model and Flavor Physics phenomena performed with data collected in proton-proton collisions at center-of-mass energies $\sqrt{s}=7 \mathrm{TeV}$ and $8 \mathrm{TeV}$. Presented results include measurements of $W$ and $Z$ boson properties as well as selected QCD phenomena. Finally, a selection of results from the $B$-physics sector is shown.
\end{abstract}

DOI:10.5506/APhysPolB.45.1505

PACS numbers: 12.10.Dm, 12.15.-y, 12.38.-t, 13.38.Be

\section{Introduction}

Following the successful start of the Large Hadron Collider (LHC) at CERN in 2009 the ATLAS detector [1] was recording data until the beginning of 2013 having collected $5.1 \mathrm{fb}^{-1}$ at the collision energy $\sqrt{s}=7 \mathrm{TeV}$ and $21.3 \mathrm{fb}^{-1}$ at $\sqrt{s}=8 \mathrm{TeV}$. This amount of experimental data allowed for detailed studies of various Standard Model processes including measurements of $W$ and $Z$ boson properties, Quantum Chromodynamics (QCD) processes as well as phenomena that include production and decays of heavy quarks. A subset of these results is presented in these paper. Following a short description of the ATLAS experimental setup in Sec. 2, Sec. 3 shows the results of selected measurements of electroweak boson properties, Sec. 4 describes recent measurements from the QCD sector, whereas Sec. 5 presents results regarding flavor physics.

* Presented at the Cracow Epiphany Conference on the Physics at the LHC, Kraków, Poland, January 8-10, 2014. 


\section{The ATLAS detector}

The ATLAS detector [1] is the largest of the four main detectors at the LHC and one of two so-called general-purpose detectors, not designed for studies of a particular branch of high-energy physics, but suitable for most extensive measurements, including Standard Model (SM) tests, Higgs boson searches, SUSY searches, and also various aspects of $B$-physics and Heavy Ion collisions. It provides almost full solid-angle coverage around the beam crossing point and allows for detailed and precise studies of collision events.

In the ATLAS coordinate system, the $z$-axis is parallel to the beam-line and points towards the anti-clockwise direction of the beam in the LHC ring. The $x$-axis points towards the ring center, setting the $\phi=0$ value of the azimuthal angle and the $y$-axis points upwards. The transverse quantities (like transverse momentum $p_{\mathrm{T}}$ or missing transverse momentum $E_{\mathrm{T}}^{\mathrm{miss}}$ ) are defined in the $x-y$ plane.

The detector is built of concentric layers around the beam-line. The innermost subsystem provides tracking of charged particles. It is immersed in a $2 \mathrm{~T}$ axial magnetic field parallel to the beam-line. Further layers consist of calorimeters and muon chambers. The muon system has its own toroidal magnetic field surrounding the detector. The Inner Detector (ID) provides coverage over the full azimuthal angle and pseudorapidity within $|\eta|<2.5$. It consists of three subsystems, the two innermost being silicon detectors, namely the Pixel and Semiconductor Tracker (SCT), and the outer being the gaseous Transition Radiation Tracker (TRT) with pseudorapidity coverage $|\eta|<2$. The calorimeters surround the ID and solenoid magnet and cover $|\eta|<4.9$. Liquid Argon (LAr) and scintillating tiles are used as active media, with lead and steel being the respective absorber materials. The calorimeters are segmented, both longitudinally and transversely, to allow for showershape studies useful for particle identification. The electromagnetic (EM) part of the ATLAS calorimeter system has a depth of at least 22 radiation lengths, whereas the hadronic part is approximately 10 interaction lengths deep. The Muon Spectrometer (MS) provides the possibility to measure muon tracks within $|\eta|<2.7$. Tracking capabilities in the MS are assured by drift tube detectors and cathode-strip chambers.

\section{3. $W$ and $Z$ boson properties}

The amount of data collected by the ATLAS detector allows for performing detailed and precise studies of the electroweak processes and comparing them to the recent results in theory. Figure 1 summarizes some of the obtained results, out of which the measurements of $W, Z$ and diboson production cross-sections are described in more details below. 


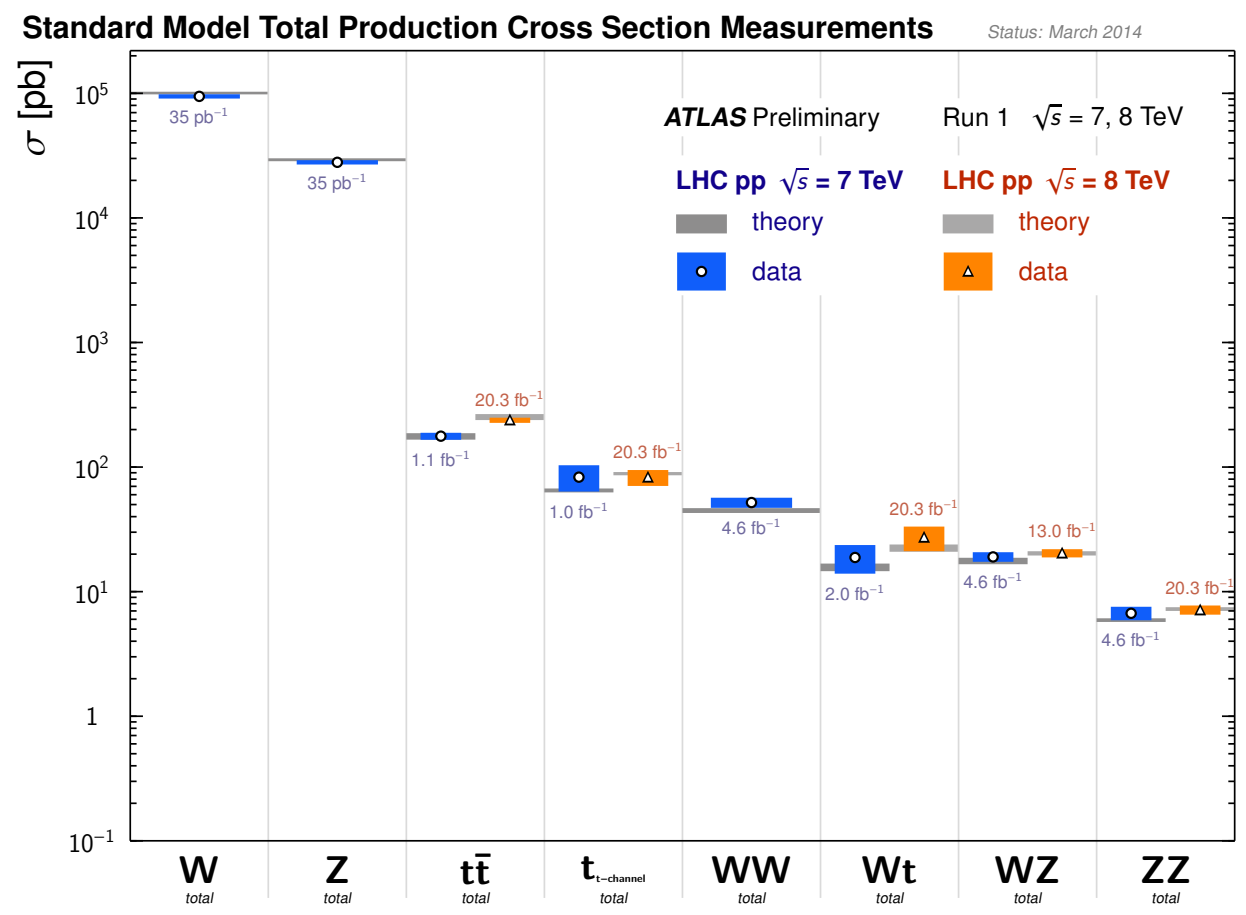

Fig. 1. Summary of a subset of Standard Model cross-sections obtained by the ATLAS Collaboration using data collected at $\sqrt{s}=7 \mathrm{TeV}$ and $8 \mathrm{TeV}$ compared to theoretical expectations [2].

\section{1. $W$ and $Z$ boson production cross-sections}

The measurements of production cross-sections of the $W$ and $Z$ bosons have been performed with the 2010 dataset (up to $36 \mathrm{pb}^{-1}$ at $7 \mathrm{TeV}$ collision energy) [3]. The resulting cross-sections are: $\sigma_{W^{ \pm}}^{\text {tot }} \times \mathrm{BR}(W \rightarrow \ell \nu)=$ $10.207 \pm 0.021$ (stat.) \pm 0.204 (syst.) \pm 0.347 (lum.) nb and $\sigma_{Z / \gamma^{*}}^{\text {tot }} \times \operatorname{BR}(Z \rightarrow$ $\ell \ell)=0.937 \pm 0.006$ (stat.) \pm 0.033 (syst.) \pm 0.032 (lum.) nb. Obtained results are in a full agreement with NNLO theory predictions. In addition, a measurement of the charge asymmetry of the $W$ bosons provides a constraint on certain Parton Density Functions (PDF) by shifting their central values at given bins and reducing uncertainties (see Fig. 2 (left)). Also, the ratio of cross-sections can be used to test Standard Model predictions (Fig. 2 (right)). 

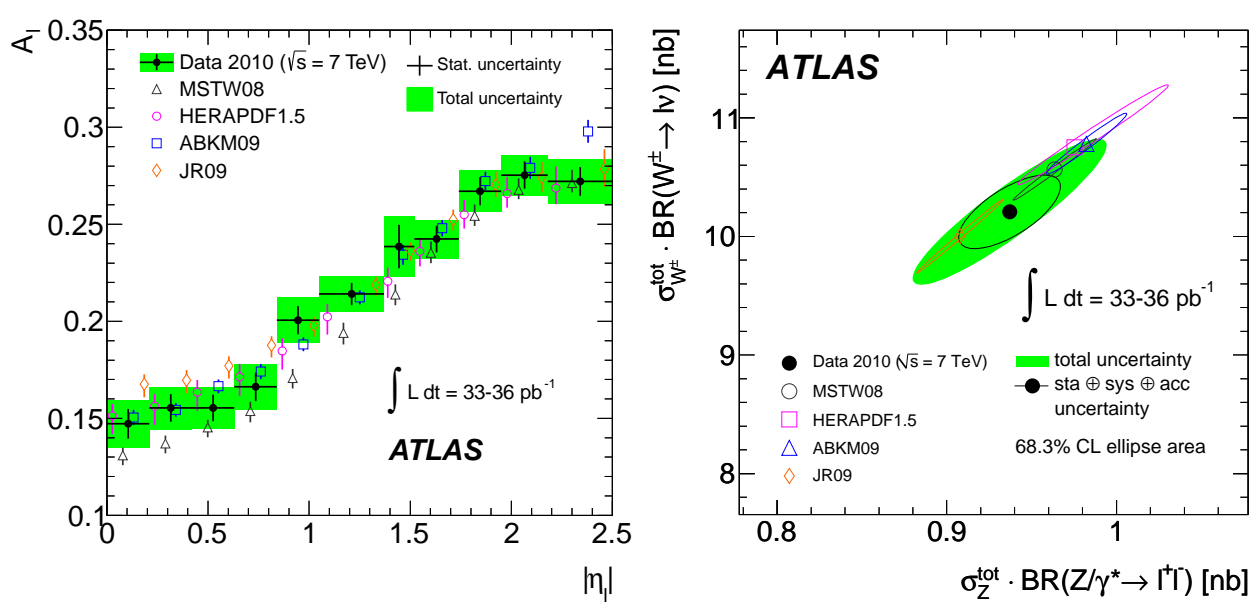

Fig. 2. Left: Measured $W$ charge asymmetry as a function of lepton pseudorapidity compared with theoretical predictions calculated to NNLO. Theoretical points are displaced for clarity within each bin. Right: Measured and predicted total crosssections times leptonic branching ratios, $\left(\sigma\left(W^{+}\right)+\sigma\left(W^{-}\right)\right)$vs. $\sigma\left(Z / \gamma^{*}\right)$. The ellipses illustrate the $68 \%$ C.L. coverage for total uncertainties (full gray/green) and excluding the luminosity uncertainty (open black). The uncertainties of the theoretical predictions correspond to the PDF uncertainties only [3].

\subsection{Forward-backward asymmetry in $Z$ decays}

Due to the $V-A$ nature of the electroweak interaction, the leptons produced in the annihilation process $q \bar{q} \rightarrow Z \rightarrow l^{+} l^{-}$present a forwardbackward asymmetry with respect to the quark direction in the rest frame of the dilepton system. The forward-backward asymmetry of lepton pairs in $Z$ boson decay, $A_{\mathrm{FB}}$ was measured using the 2011 dataset of $4.7 \mathrm{fb}^{-1}$ collected at $\sqrt{s}=7 \mathrm{TeV}$ [4]. An example of its behavior for central-forward electron pairs with respect to their invariant mass is shown in Fig. 3 (left). This quantity is sensitive to weak mixing angle, $\sin ^{2} \theta_{W}^{\text {eff }}$, which can be extracted from the measurement. The combination of the muon and electron channels yields a value of $\sin ^{2} \theta_{W}^{\text {eff }}=0.2297 \pm 0.0004$ (stat.) \pm 0.0009 (syst). The comparison of this value with previous results from other experiments can be seen in Fig. 3 (right). 

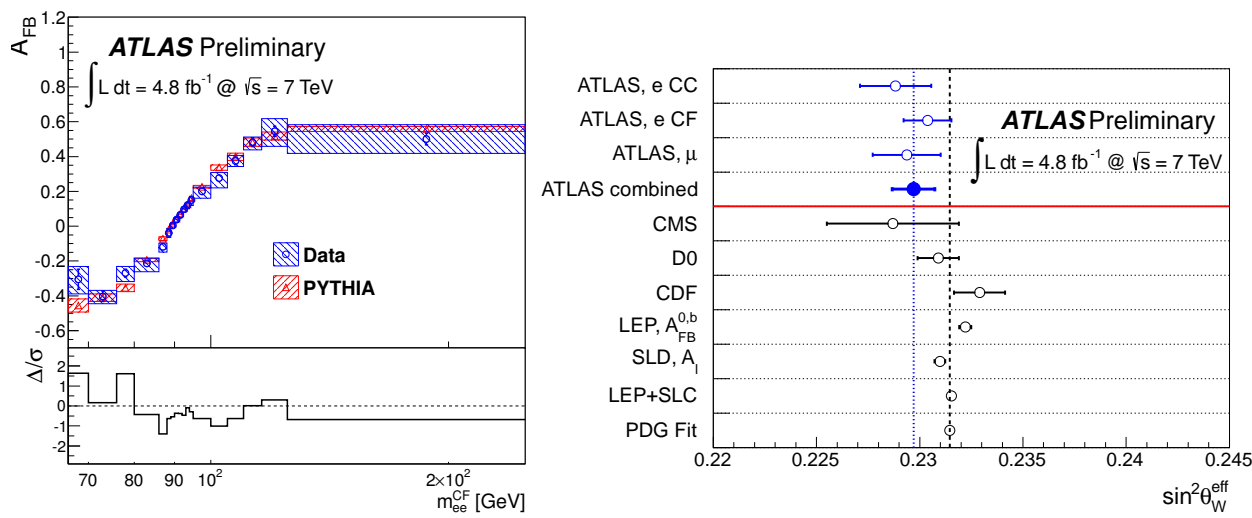

Fig. 3. Left: $A_{\mathrm{FB}}$ unfolded to Born-level for central-forward electron pairs. For the data, the boxed shaded region represents the total (statistical+systematic) uncertainty and the error bars represent the statistical uncertainty. The boxed shaded regions for the MC represent only the statistical uncertainty. Right: Comparison of the results of this analysis with other published results for weak mixing angle. A vertical dotted line illustrates the results from the ATLAS combined measurement reported here and a vertical dashed line shows the results from the current PDG global fit [4].

\section{3. $Z+$ jets production}

The production of jets in association with a $Z$ boson at hadron colliders provides an important test of perturbative quantum chromodynamics (pQCD). Such processes also constitute a non-negligible background for studies of the Higgs boson candidate. Inclusive and differential jet crosssections in $Z$ events, with $Z$ decaying into electron or muon pairs, are measured for jets with transverse momentum $p_{\mathrm{T}}>30 \mathrm{GeV}$ and rapidity $|y|<4.4$. The results are compared to next-to-leading-order perturbative QCD calculations, and to predictions from different Monte Carlo generators based on leading-order and next-to-leading-order matrix elements supplemented by parton showers [5]. Such measurement allows checking theoretical predictions for jet multiplicities and momenta. Measured cross-section for $Z+$ jets production as a function of jet multiplicity is shown in Fig. 4 (left), whereas ratios of cross-sections for successive jet multiplicities can be seen in Fig. 4 (right). 

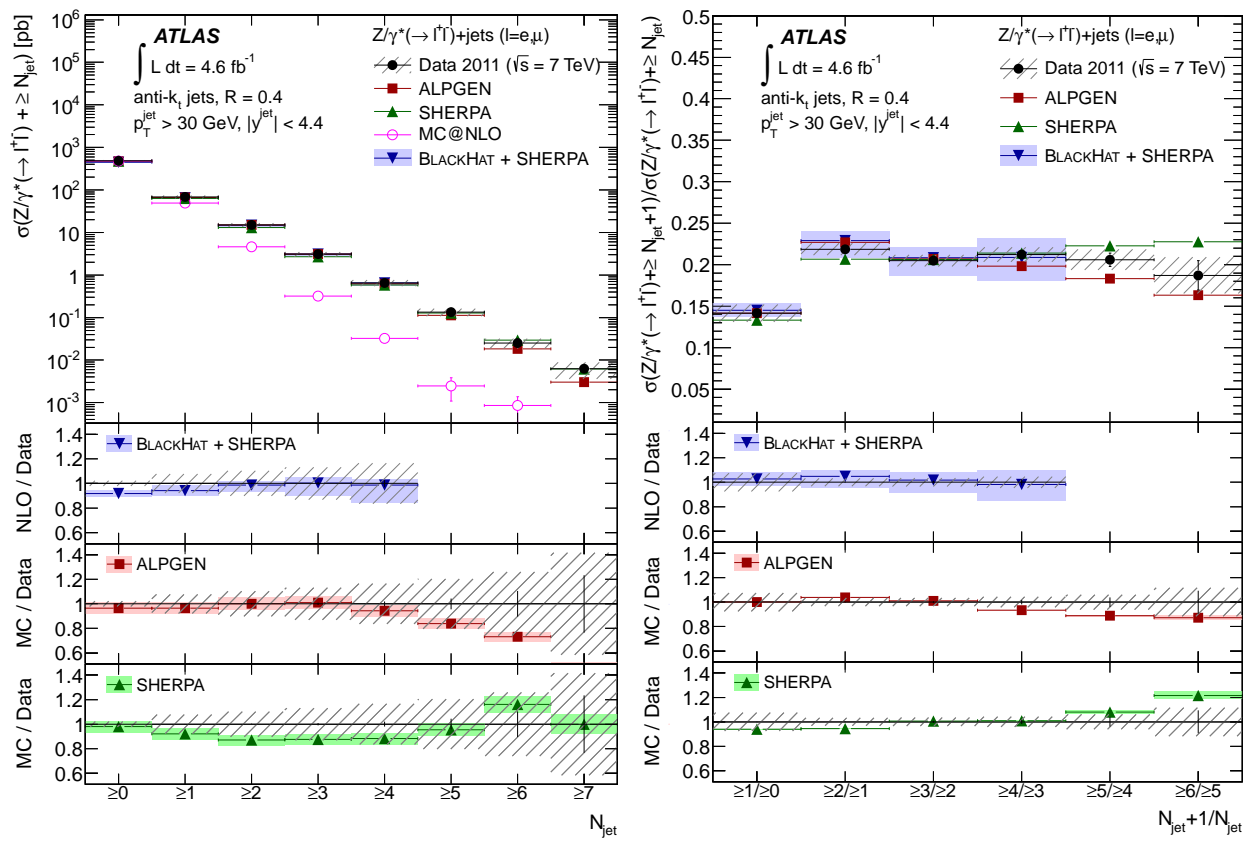

Fig. 4. Measured cross-section for $Z(\rightarrow l l)+$ jets as a function of the inclusive jet multiplicity, $N_{\text {jet }}$ (left), and ratio of cross-sections for successive inclusive jet multiplicities (right). The data are compared to NLO pQCD predictions from BLACKHAT+SHERPA corrected to the particle level, and the ALPGEN, SHERPA and MC@NLO (as in the legend) event generators [5].

\section{4. $W+$ charm production}

The production of a $W$ boson in association with a single charm quark is studied using $4.6 \mathrm{fb}^{-1}$ of $p p$ collision data at $\sqrt{s}=7 \mathrm{TeV}$. In events in which a $W$ boson decays to an electron or muon, the charm quark is tagged either by its semileptonic decay to a muon or by the presence of a charmed meson. The integrated and differential cross-sections as a function of pseudorapidity of the lepton from the $W$-boson decay are measured. Results are compared to the predictions of next-to-leading-order QCD calculations obtained from various parton distribution function parameterisations. The ratio of the strange-to-down sea-quark distributions is determined to be 0.96 \pm 0.30 at $Q^{2}=1.9 \mathrm{GeV}^{2}$, which supports the hypothesis of $\mathrm{SU}(3)$-symmetric composition of the light-quark sea. Additionally, the cross-section ratio $\sigma\left(W^{+}+\bar{c}\right) / \sigma\left(W^{-}+c\right)$ is compared to predictions obtained using parton distribution function parameterisations with different assumptions about the $s \bar{s}$ quark asymmetry [6]. The plots in Fig. 5 show the aforementioned ratio (left) and asymmetry (right). 

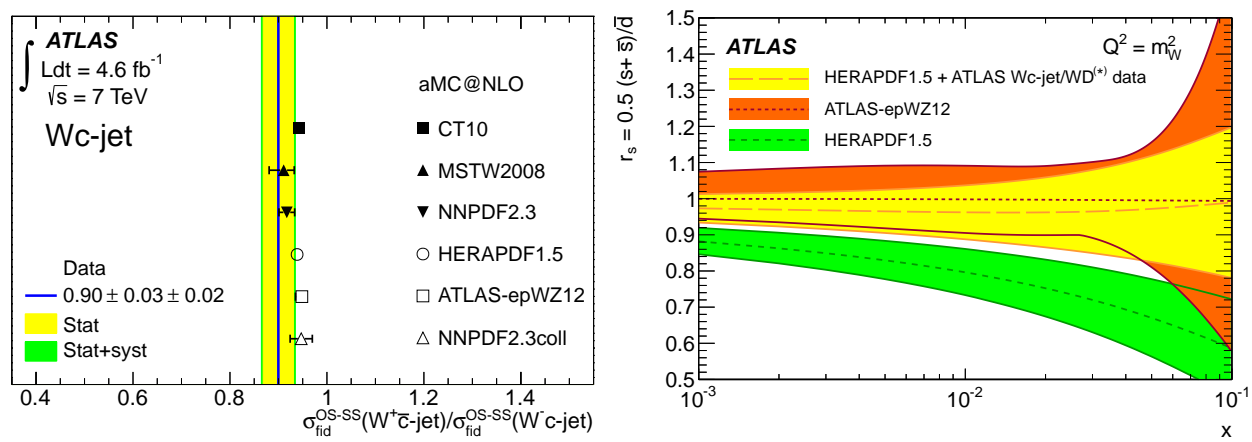

Fig. 5. Left: Ratio of $\sigma\left(W^{+}+\bar{c}\right) / \sigma\left(W^{-}+c\right)$ resulting from the averaging procedure compared to various PDF predictions based on aMC@NLO. Right: Ratio of strangeto-down sea-quark distributions $r_{\mathrm{s}}=0.5(s+\bar{s}) / \bar{d}$ as a function of $x$ as assumed in HERAPDF1.5 PDF compared to the ratio obtained from the fit including the ATLAS $W c$-jet $/ W D^{(*)}$ data and the ratio obtained from ATLAS-epWZ12 [6].

\subsection{High-mass Drell-Yan processes}

The cross-section for the Drell-Yan (DY) process was measured using $4.9 \mathrm{fb}^{-1}$ of data at $\sqrt{s}=7 \mathrm{TeV}$ in the mass region between 116 and $1500 \mathrm{GeV}$ reaching beyond the $Z$ pole. This analysis was conducted in $Z / \gamma^{*} \rightarrow e e$ de-
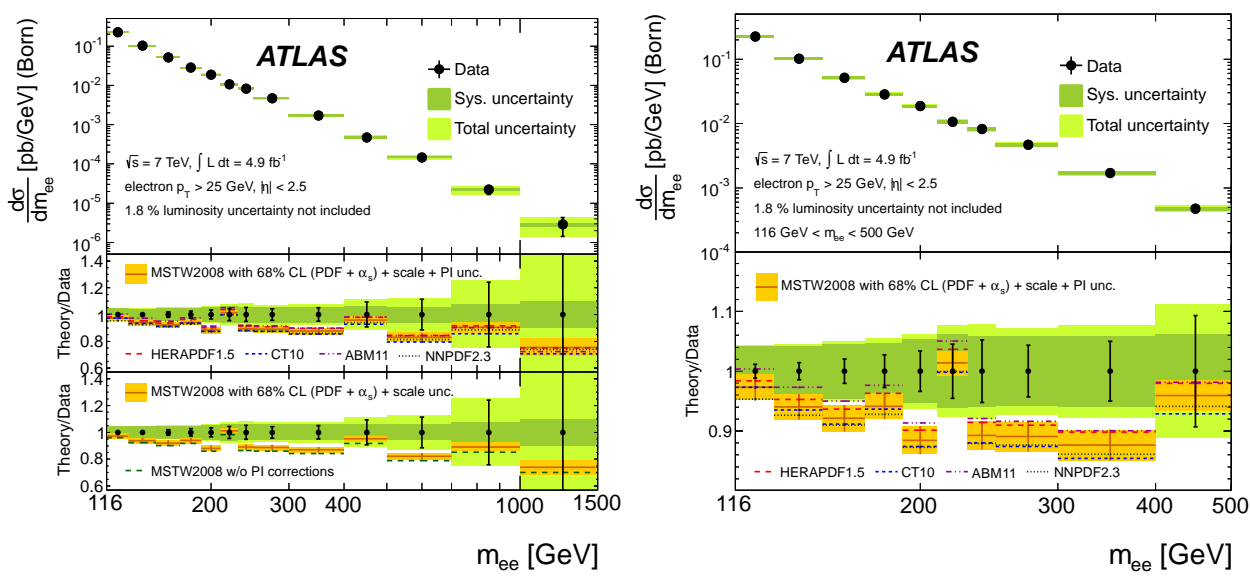

Fig. 6. Measured differential cross-section at the Born level within the fiducial region (electron $p_{\mathrm{T}}>25 \mathrm{GeV}$ and $|\eta|<2.5$ ) with statistical, systematic, and combined statistical and systematic (total) uncertainties, excluding the $1.8 \%$ uncertainty on the luminosity. The measurement is compared to FEWZ 3.1 calculations at NNLO QCD with NLO electroweak corrections using the $G_{\mu}$ electroweak parameter scheme [7]. 
cay channel. Given the low background, a small experimental uncertainty can be achieved on the measured invariant mass distribution allowing for a precision test of $\mathrm{pQCD}$. The mass spectrum is also sensitive to PDFs, in particular to the poorly known distribution of antiquarks at large $x$. Additionally, the production of DY dilepton pairs is a source of background for other Standard Model measurements, and the mass spectrum may be modified by New Physics phenomena giving rise to, e.g., narrow resonances or an excess of high-mass pairs inconsistent with the known PDFs. The plots presented in Fig. 6 show the differential cross-sections for Drell-Yan process versus the invariant mass of electron pair in various ranges [7].

\subsection{Diboson production}

The diboson production probes Triple-Gauge Couplings (TGC) - a possible manifestation of New Physics in neutral TGC's $(Z Z \gamma, Z Z Z$ and $Z \gamma \gamma)$ which are not allowed in SM. It also constitutes a significant irreducible background to Higgs measurements (in $W W$ and $Z Z$ channels). The ATLAS experiment has conducted such measurements with the datasets totaling $13-21 \mathrm{fb}^{-1}$ collected at $\sqrt{s}=8 \mathrm{TeV}$. The measured cross-section for double$Z$ boson production is $\sigma_{Z Z}^{\text {tot }}=7.1 \pm 0.5$ (stat.) \pm 0.3 (syst.) \pm 0.2 (lumi.) pb [8] and the cross-section for $W Z$ production is $\sigma_{W Z}^{\text {tot }}=20.3 \pm 0.8$ (stat.) \pm 1.2 (syst.) \pm 0.7 (lumi.) pb [9]. The obtained results are well within the Standard Model expectations as can be seen in Fig. 7.
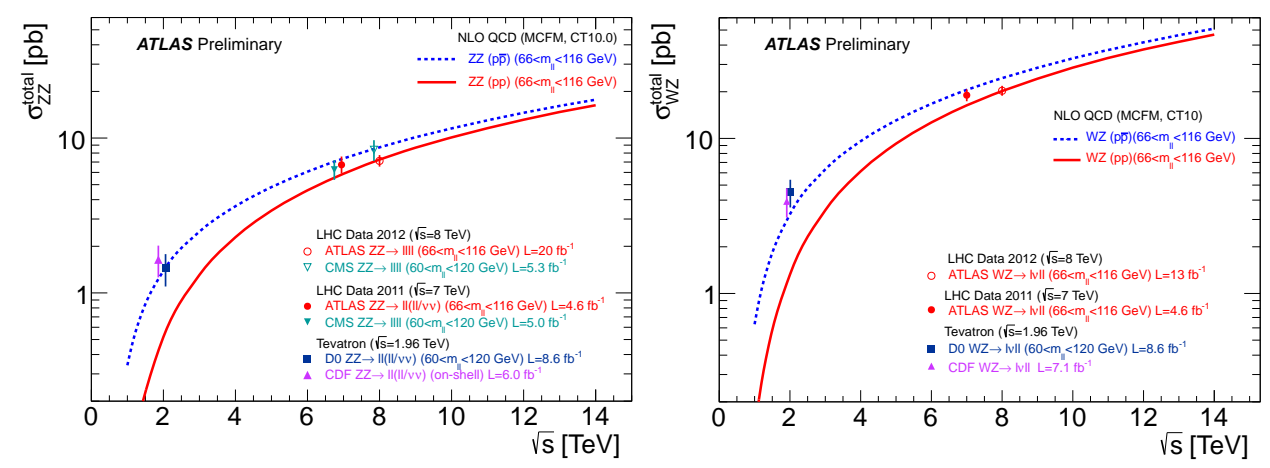

Fig. 7. Comparison of experimental measurements and theoretical predictions of the total $Z Z$ (left) [8] and $W Z$ (right) [9] production cross-section as a function of center-of-mass energy. Shown are experimental measurements from CDF and D0 in $p \bar{p}$ collisions at the Tevatron at $\sqrt{s}=1.96 \mathrm{TeV}$, and experimental measurements from ATLAS and CMS in $p p$ collisions at the LHC at $\sqrt{s}=7 \mathrm{TeV}$ and $\sqrt{s}=8 \mathrm{TeV}$. 


\section{QCD results}

The ATLAS experiment is a perfect device for precise studies of the nature of strong interactions. The highest available collision energy as well as enormous statistics of collected data allow for detailed tests of the predictions of QCD regarding the nature of phenomena occurring in hadronic collisions.

\subsection{Inclusive jet cross-section}

A direct probe of QCD in its perturbative regime is provided by the measurement of inclusive jet cross-section. This measurement probes also the PDFs of protons. The ATLAS Collaboration has performed this measurement with 2010 data $\left(37 \mathrm{pb}^{-1}\right)$ collected at $\sqrt{s}=7 \mathrm{TeV}$. Results on double-differential cross-section of inclusive jet production versus transverse momentum and rapidity show a good agreement with QCD predictions up to very high transverse momenta $(1.5 \mathrm{TeV})$, as seen in Fig. 8 [10].

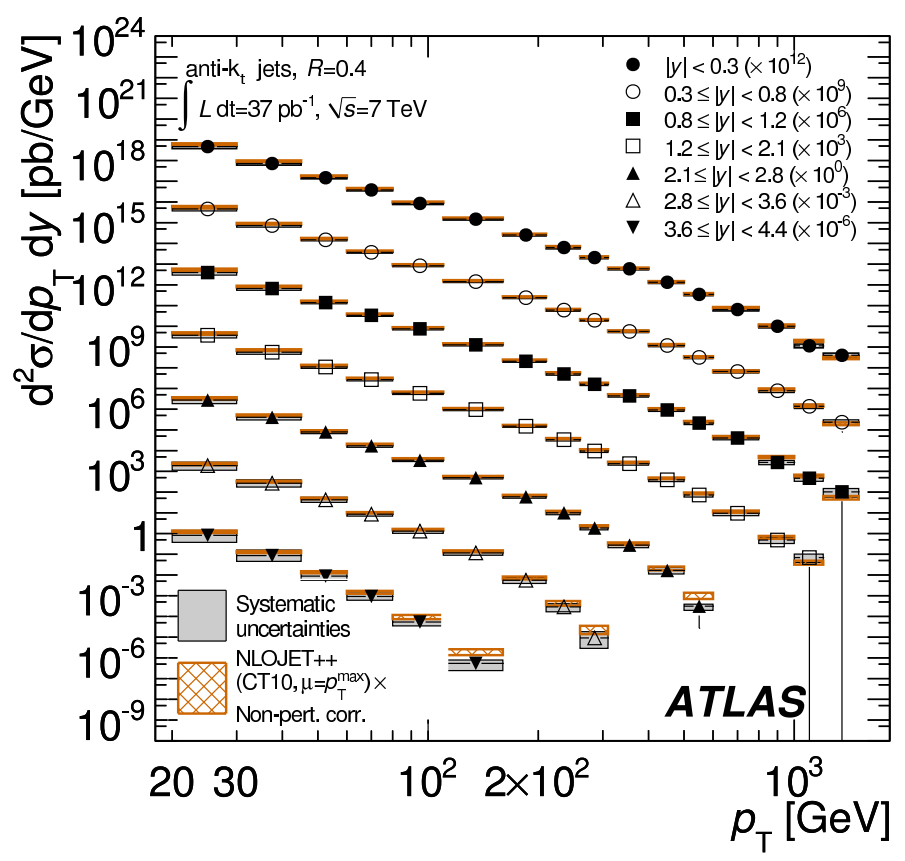

Fig. 8. Inclusive jet double-differential cross-sections as a function of jet $p_{\mathrm{T}}$ in different regions of $|y|$ for jets identified using the anti- $k_{\mathrm{t}}$ algorithm with $R=0.4$. For convenience, the cross-sections are multiplied by the factors indicated in the legend. The data are compared to NLO pQCD calculations using NLOJET++ to which non-perturbative corrections have been applied [10]. 


\subsection{Jet cross-section ratio}

The ATLAS experiment has also collected data in proton-proton collisions at $\sqrt{s}=2.76 \mathrm{TeV}$ having recorded $0.2 \mathrm{pb}^{-1}$ of integrated luminosity in 2011. The ratio of the cross-section to the inclusive jet cross-section measurement at $\sqrt{s}=7 \mathrm{TeV}$ is calculated as a function of both transverse momentum and the dimensionless quantity $x_{\mathrm{T}}=2 p_{\mathrm{T}} / \sqrt{s}$, in bins of jet rapidity. The systematic uncertainties on the ratios are significantly reduced due to the cancellation of correlated uncertainties in the two measurements [11]. Figure 9 (left) shows the measured cross-section ratio as a function of jet $p_{\mathrm{T}}$ in bins of jet rapidity. The impact of this measurement on the PDFs is presented in Fig. 9 (right).
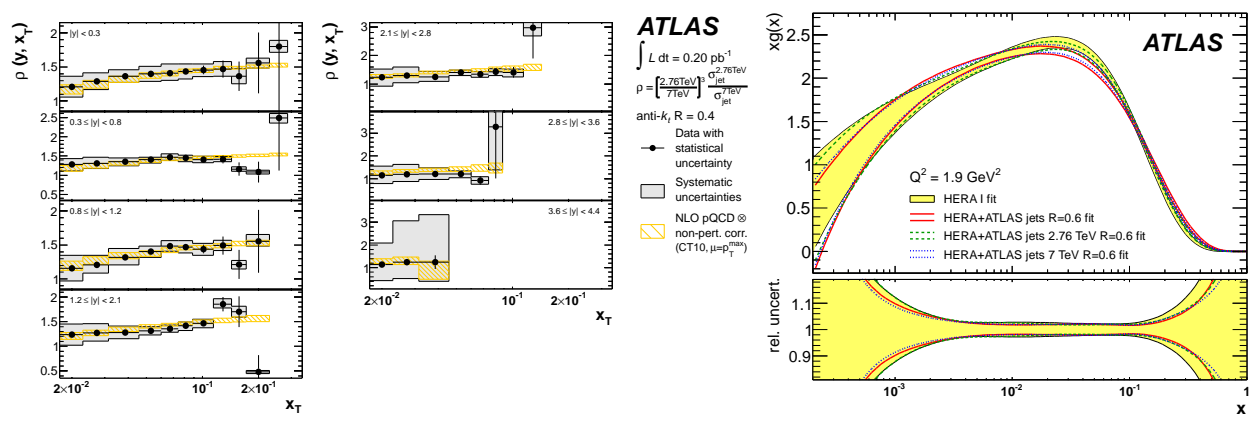

Fig. 9. Left: Ratio of the inclusive jet cross-section at $\sqrt{s}=2.76 \mathrm{TeV}$ to the one at $\sqrt{s}=7 \mathrm{TeV}$ as a function of $x_{\mathrm{T}}$ in bins of jet rapidity, for anti- $k_{\mathrm{t}}$ jets with $R=0.4$. Right: Momentum distributions of the gluon $x g(x)$ together with their relative experimental uncertainty as a function of $x$ for $Q^{2}=1.9 \mathrm{GeV}^{2}$. The filled area indicates a fit to HERA data only. The bands show fits to HERA data in combination with both ATLAS jet datasets, and with the individual ATLAS jet datasets separately [11].

\subsection{Running of $\alpha_{\mathrm{s}}$}

In the regime of perturbative $\mathrm{QCD}$, the number of events with additional radiated parton is proportional to the value of the strong coupling constant, $\alpha_{\mathrm{S}}$. It is expected that this behavior can be also observed by measuring the ratio of cross-sections of events with $N+1$ and $N$ jets. In the presented case, the ATLAS experiment has measured the cross-section ratio of events with 3 jets to events with 2 jets with respect to jet transverse momentum (see Fig. 10 (left)) [12]. The extracted value of the strong coupling constant at the $Z$-pole is in agreement with the SM expectation, $\alpha_{\mathrm{S}}\left(M_{\mathrm{Z}}\right)=0.111 \pm 0.006$ (exp.) \pm 0.016 (theory). The running of $\alpha_{\mathrm{S}}$ from several measurements including the presented one is shown in Fig. 10 (right). 

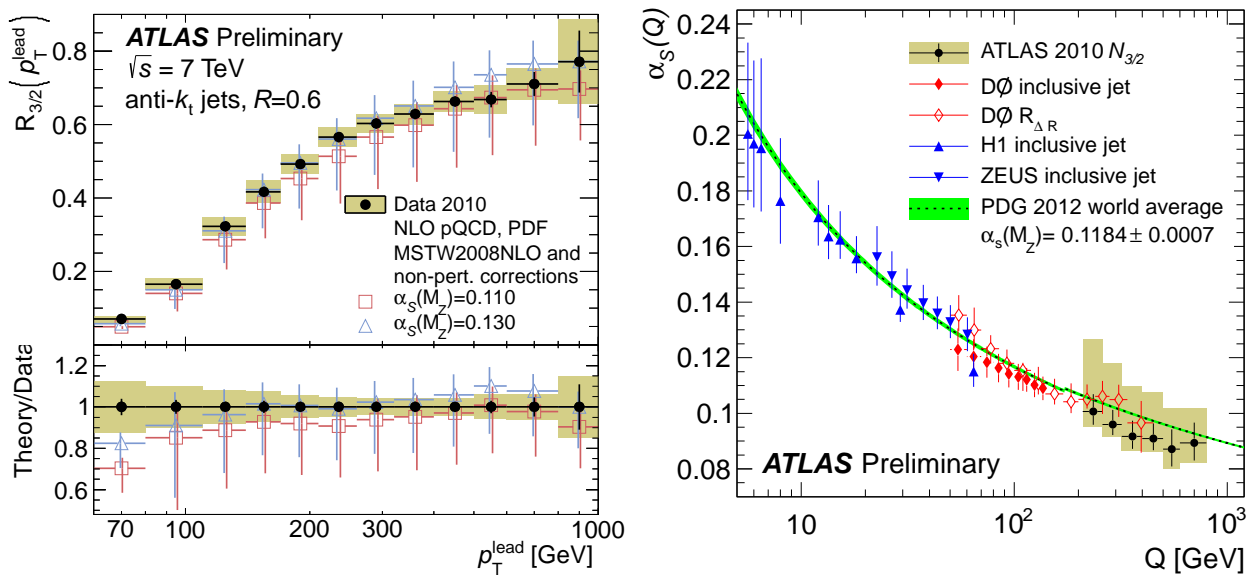

Fig. 10. Left: Ratio of cross-sections of three to two-jet events versus transverse momentum of the leading jet. Right: Strong coupling constant extracted from several measurements as a function of the renormalization scale $Q$ [12].

\subsection{Inclusive photon cross-section}

Prompt photon production at hadron colliders allows precise tests of perturbative QCD predictions by providing a colorless probe of the hard scattering process. The measurement is sensitive to the gluon content of the
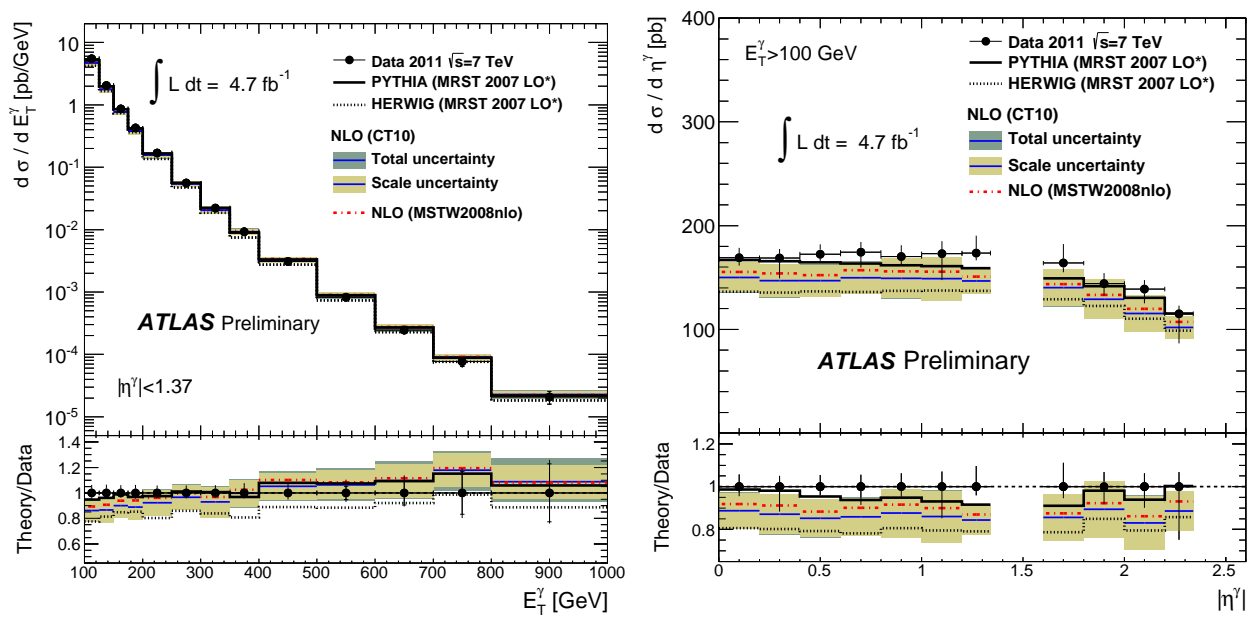

Fig. 11. Left: Measured (dots with error bars) and expected inclusive prompt photon cross-section in the $|\eta|<1.37$ region. Right: Measured and expected inclusive prompt photon cross-section as a function of the absolute value of $\eta$, for photons with transverse energies above $100 \mathrm{GeV}$ excluding $1.37<|\eta|<1.52$ [13]. 
proton through the $q g \rightarrow q \gamma$ process, which dominates the prompt photon production cross-section at the LHC, and can thus be used to constrain parton distribution functions. The ATLAS Collaboration has measured production cross-section of isolated prompt photons in the transverse energy region of $100 \mathrm{GeV}<E_{\gamma}^{\mathrm{T}}<1 \mathrm{TeV}$ in the 2011 dataset of $4.7 \mathrm{fb}^{-1}$ collected at $\sqrt{s}=7 \mathrm{TeV}$ [13]. The comparison of measured differential cross-sections to perturbative QCD predictions at NLO shows good agreement, see Fig. 11.

\section{Results from flavor physics sector}

The goals of the heavy-flavor physics program at ATLAS are to test theoretical models for heavy-flavor production within the Standard Model and to search for New Physics through rare decays or new sources of CP violation. The proceedings present a selection of analyses that were completed with the data sample of about $4.7 \mathrm{fb}^{-1}$ collected in 2011 with $\sqrt{s}=7 \mathrm{TeV}$.

\subsection{Production of $\chi_{\mathrm{c}_{1}}$ and $\chi_{\mathrm{c}_{2}}$}

The observation of production of charmonium excited states, $\chi_{\mathrm{c}_{1}}$ and $\chi_{\mathrm{c}_{2}}$, provides complementary information about charmonium production to the observations of $J / \psi$ and $\psi(2 S)$. The $\chi_{\mathrm{c}_{1}}$ and $\chi_{\mathrm{c}_{2}}$ are produced either in a prompt mode or in a decay chain of a $b$-hadron. Observation of these excited states is possible via selecting the $\chi_{\mathrm{c}_{1,2}} \rightarrow J / \psi\left(\mu^{+} \mu^{-}\right) \gamma\left(e^{+} e^{-}\right)$and placing cuts on the mass difference between $m\left(\mu^{+} \mu^{-} \gamma\right)-m\left(\mu^{+} \mu^{-}\right)$[14]. The measured differential cross-section of excited charmonium states versus transverse momentum of the $J / \psi$ meson can be seen in Fig. 12 (left: $\chi_{\mathrm{c}_{1}}$, right: $\left.\chi_{\mathrm{c}_{2}}\right)$.
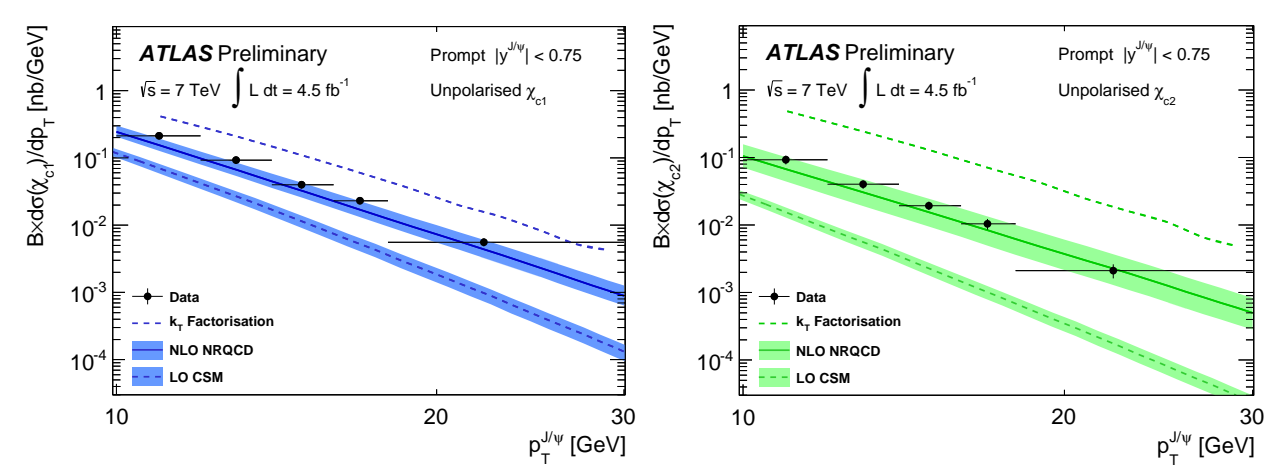

Fig. 12. Measured differential cross-section for $\chi_{\mathrm{c}_{1}}$ (left) and $\chi_{\mathrm{c}_{2}}$ (right) with respect to $p_{\mathrm{T}}$ of the $J / \psi$ meson [14]. 


\subsection{Production of $J / \psi$ in association with $W$ bosons}

The process $p p \rightarrow W^{ \pm} J / \psi$ provides a powerful probe of the production mechanism of charmonium in hadronic collisions, and is also sensitive to multiple parton interactions in the colliding protons. The ATLAS Collaboration has performed first observation of the production of $W^{ \pm}$in association with prompt $J / \psi$ events in hadronic collisions having found a total of $27.4_{-6.5}^{+7.5}$ events with statistical significance of $5.1 \sigma$ [15]. The analysis is performed by selecting events with at least three muons, two of which have to form a $J / \psi$ candidate and the third one is combined with the transverse missing energy (accounting for neutrino) to form a $W^{ \pm}$candidate. An unbinned maximum likelihood fit in $J / \psi$ candidate invariant mass and pseudo-proper time is used to obtain yields for prompt $J / \psi$, non-prompt $J / \psi$ and prompt/non-prompt combinatoric backgrounds. Results of this fit can be seen in Fig. 13.
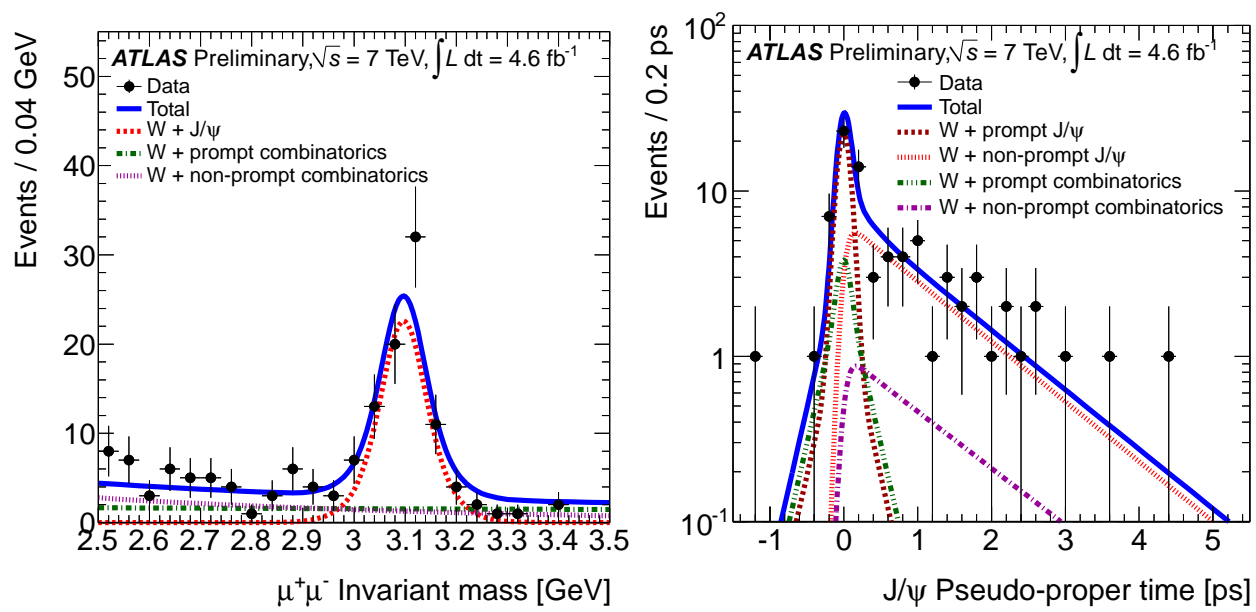

Fig. 13. Projections in invariant mass (left) and pseudo-proper time (right) of the two-dimensional mass-pseudo-proper time fit used to extract the prompt $\mathrm{J} / \psi$ candidates [15].

\subsection{Angular analysis of $B_{s}^{0} \rightarrow J / \psi \Phi$}

A measurement of the $B_{s}^{0} \rightarrow J / \psi \phi$ decay parameters, updated to include flavor tagging has been performed in the ATLAS experiment. The values measured for the physical parameters are:

$$
\begin{aligned}
\phi_{s} & =0.12 \pm 0.25 \text { (stat.) } \pm 0.11 \text { (syst.) } \mathrm{rad}, \\
\Delta \Gamma_{s} & =0.053 \pm 0.021 \text { (stat.) } \pm 0.009 \text { (syst.) } \mathrm{ps}^{-1}, \\
\Gamma_{s} & =0.677 \pm 0.007 \text { (stat.) } \pm 0.003 \text { (syst.) } \mathrm{ps}^{-1}, \\
\left|A_{0}(0)\right|^{2} & =0.529 \pm 0.006 \text { (stat.) } \pm 0.011 \text { (syst.), }
\end{aligned}
$$




$$
\begin{aligned}
\left|A_{\|}(0)\right|^{2} & =0.220 \pm 0.008 \text { (stat.) } \pm 0.009 \text { (syst.) } \\
\delta_{\perp} & =3.89 \pm 0.46 \text { (stat.) } \pm 0.13 \text { (syst.) rad }
\end{aligned}
$$

where the parameter $\Delta \Gamma_{s}$ is constrained to be positive. The fraction $\left|A_{S}(0)\right|^{2}$ of $S$-wave $K K$ or $f_{0}$ contamination through the decays $B_{s} \rightarrow J / \psi K^{+} K^{-}\left(f_{0}\right)$ is also measured and is found to be compatible with zero [16].

\subsection{Angular analysis of $B_{d}^{0} \rightarrow K^{* 0} \mu^{+} \mu^{-}$}

The process $B_{d}^{0} \rightarrow K^{* 0} \mu^{+} \mu^{-}$contains quark transition $b \rightarrow s$ which, in the Standard Model, is only possible via loops. This analysis is therefore sensitive to possible New Physics contributions and allows for extraction of the $K^{* 0}$ longitudinal momentum fraction $F_{\mathrm{L}}$ and lepton pair forward-backward asymmetry $A_{\mathrm{FB}}$ (see Fig. 14) with a sequential fit of $B_{d}^{0}$ invariant mass and three decay angles [17]. The results are consistent with SM expectations and the uncertainty is dominated by statistics. The analysis of the full dataset of about $20 \mathrm{fb}^{-1}$ from 2012 is ongoing.
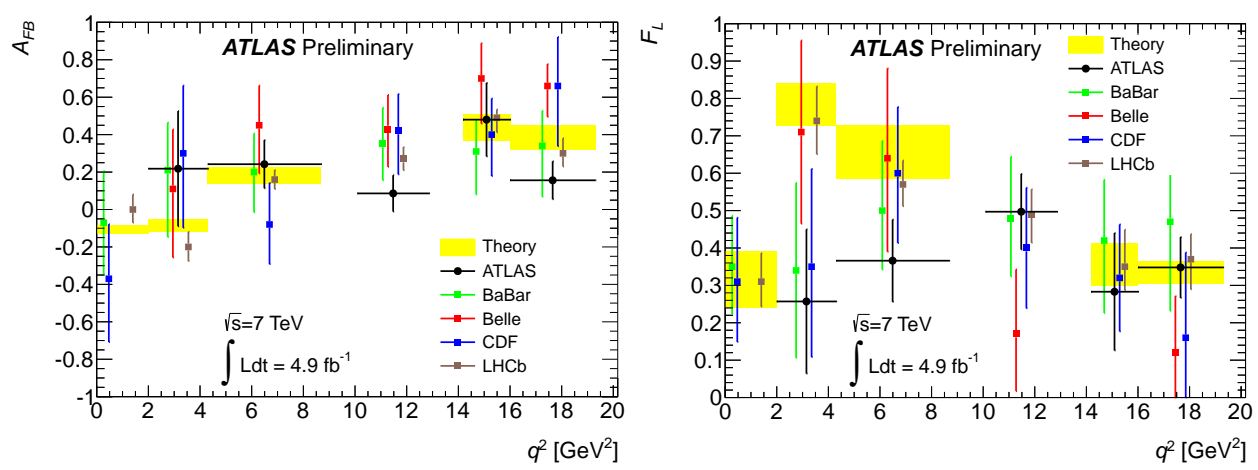

Fig. 14. Forward-backward asymmetry $A_{\mathrm{FB}}$ (left) and fraction of longitudinal polarized $K^{* 0}$ mesons $F_{\mathrm{L}}$ (right) as a function of $q^{2}$ measured by ATLAS (black dots). In each $q^{2}$ bin, results of other experiments are shown as colored squares: BaBar, Belle, CDF and LHCb [17].

\subsection{Parity violation in $\Lambda_{b} \rightarrow J / \psi \Lambda^{0}$}

A measurement of the parity violating decay asymmetry parameter, $\alpha_{b}$, and the helicity amplitudes for the $\Lambda_{b}^{0}$ hyperon decay $\Lambda_{b}^{0} \rightarrow J / \psi(\mu \mu) \Lambda^{0}\left(p \pi^{-}\right)$ has been performed in the ATLAS experiment. The analysis is based on about $1400 \Lambda_{b}^{0}$ and $\bar{\Lambda}_{b}^{0}$ baryons selected in $4.6 \mathrm{fb}^{-1}$ of proton-proton collision data. By combining the $\Lambda_{b}^{0}$ and $\bar{\Lambda}_{b}^{0}$ samples under the assumption of CP conservation, the value of $\alpha_{b}$ was measured to be $0.30 \pm 0.16$ (stat.) \pm 0.06 (syst.). This measurement provides a test of several theoretical models that predict $\alpha_{b}$ values ranging from -0.2 to +0.8 [18]. 


\section{Summary}

The presented results form a small subset of many analyses that have been conducted by the ATLAS Collaboration concerning the Standard Model processes including the physics of electroweak bosons, soft QCD and heavy flavor sector. Excellent performance of both the Large Hadron Collider and ATLAS experimental setup allowed for many precise tests of the Standard Model expectations and extrapolations from lower-energy experiments.

This work was supported in part by the Polish National Science Center grants DEC-2013/08/M/ST2/00320 and 2012/07/B/ST2/03680, and by PL-Grid Infrastructure.

\section{REFERENCES}

[1] ATLAS Collaboration, JINST 3, S08003 (2008).

[2] Summary plots from the ATLAS Standard Model physics group: https://atlas.web.cern.ch/Atlas/GROUPS/PHYSICS/ CombinedSummaryPlots/SM/

[3] ATLAS Collaboration, Phys. Rev. D85, 072004 (2012).

[4] ATLAS Collaboration, ATLAS-CONF-2013-043, http://cdsweb. cern. ch/record/1544035

[5] ATLAS Collaboration, J. High Energy Phys. 07, 032 (2013).

[6] ATLAS Collaboration, arXiv:1402.6263 [hep-ex], submitted to J. High Energy Phys.

[7] ATLAS Collaboration, Phys. Lett. B725, 223 (2013).

[8] ATLAS Collaboration, ATLAS-CONF-2013-020, http://cdsweb. cern. ch/record/1525555

[9] ATLAS Collaboration, ATLAS-CONF-2013-021, http://cdsweb. cern.ch/record/1525557

[10] ATLAS Collaboration, Phys. Rev. D86, 014022 (2012).

[11] ATLAS Collaboration, Eur. Phys. J. C73, 2509 (2013).

[12] ATLAS Collaboration, ATLAS-CONF-2013-041, http://cdsweb. cern. ch/record/1543225

[13] ATLAS Collaboration, ATLAS-CONF-2013-022, http://cdsweb. cern. ch/record/1525723

[14] ATLAS Collaboration, ATLAS-CONF-2013-095, http://cdsweb. cern. ch/record/1596292

[15] ATLAS Collaboration, arXiv:1401.2831 [hep-ex], submitted to J. High Energy Phys. 
[16] ATLAS Collaboration, ATLAS-CONF-2013-039, http: //cdsweb. cern. ch/record/1541823

[17] ATLAS Collaboration, ATLAS-CONF-2013-038, http://cdsweb. cern.ch/record/1537961

[18] ATLAS Collaboration, arXiv:1404.1071 [hep-ex], submitted to Phys. Rev. D. 